\title{
Electric Conductivity, Elasticity, and Optical Spectra in Blend Gels of Polydiacetylenes: P(3BCMU)/P(4BCMU)/Mixed Solvent System
}

\author{
Pingfan Chen, ${ }^{\dagger}$ Keiichiro Adachi, ${ }^{*}$ and Tadao Kotaka \\ Department of Macromolecular Science, Faculty of Science, \\ Osaka University, Toyonaka, Osaka 560, Japan
}

(Received August 24, 1992)

\begin{abstract}
Alternating current conductivity $\sigma_{\mathrm{ac}}$, shear moduli $G^{\prime}$, and ultraviolet-spectra (UV) were studied for gels consisting of two polydiacetylenes i.e., poly[4,6-decadiyn-1,10-diol-bis $(n$ butoxy-carbonylmethyl urethane)] abbreviated as $\mathrm{P}(3 \mathrm{BCMU})$ and poly[5,7-dodecadiyn-1,12-diolbis( $n$-butoxy carbonyl methylurethane)] abbreviated as $\mathrm{P}(4 \mathrm{BCMU})$. As a common solvent of these polydiacetylenes, a mixed solvent $p$-dichlorobenzene ( $p$-DCB)/toluene (TOL) $(62 / 38)$ was used. Shear moduli $G^{\prime}$ of blend gels decreased stepwise around 340 and $385 \mathrm{~K}$ which coincided with the gel-to-sol transition temperatures of pure $\mathrm{P}(4 \mathrm{BCMU})$ and $\mathrm{P}(3 \mathrm{BCMU})$ gels, respectively. At $385 \mathrm{~K}$ the blend gels transformed completely into sol. Observed $G^{\prime}$ of blend gels conformed approximately to the additivity law with respect to the weight fraction of $\mathrm{P}(3 \mathrm{BCMU})$ and $\mathrm{P}(4 \mathrm{BCMU})$. This indicates that in the range of $T<340$ the gel network is composed of both the $\mathrm{P}(4 \mathrm{BCMU})$ and $\mathrm{P}(3 \mathrm{BCMU})$ molecules but above $340 \mathrm{~K}$ the network is composed only of the $\mathrm{P}(3 \mathrm{BCMU})$ molecules. Temperature dependence of $\sigma_{\mathrm{ac}}$ at $1 \mathrm{kHz}$ exhibited two maxima around 330 and $360 \mathrm{~K} . \sigma_{\mathrm{ac}}$ observed around $360 \mathrm{~K}$ was $c a$. ten times higher than $\sigma_{\mathrm{ac}}$ expected from the additivity. The UV spectra of the blend gels exhibited abosorption bands not seen in pure gels of the components. It is speculated that the $\mathrm{P}(3 \mathrm{BCMU})$ and $\mathrm{P}(4 \mathrm{BCMU})$ chains partially form a charge transfer complex in blend gels.

KEY WORDS Gel / Electric Conductivity / Elasticity / UV Spectra / Blend

/ Polydiacetylene / P(3BCMU) / P(4BCMU) / Gel-to-Sol Transition
\end{abstract}

In our previous papers, ${ }^{1-3}$ we reported the electric conductivity, sol-gel transition behavior and ultraviolet and visible (UV) spectra in gels of polydiacetylenes. Specifically they were poly[4,6-decadiyn-1,10-diol-bis $(n$ butoxy-carbonylmethyl urethane)] abbreviated as $\mathrm{P}(3 \mathrm{BCMU})$ and poly[5,7-dodecadiyn1,12-diol-bis( $n$-butoxy carbonyl methylurethane)] abbreviated as $\mathrm{P}(4 \mathrm{BCMU})$. These polydiacetylens are soluble in a variety of solvents and form gels in poor solvents such as $o$-dichlorobenzene (o-DCB) and toluene (TOL) at concentration as low as $0.1 \mathrm{wt} \% .^{4,5}$ The electronic conduction in $\mathrm{P}(3 \mathrm{BCMU})$ and $\mathrm{P}(4 \mathrm{BCMU})$ cast films, ${ }^{6,7}$ and that in the single crystal of $\mathrm{P}(4 \mathrm{BCMU})^{8}$ were studied throughly but the conduction behavior and physical properties in the gel state have not been studied fully.

Although the structures of the side groups of $\mathrm{P}(3 \mathrm{BCMU})$ and $\mathrm{P}(4 \mathrm{BCMU})$ are similar their properties differ greatly. ${ }^{1-3,9}$ For example, the color of $\mathrm{P}(4 \mathrm{BCMU})$ gel is red but that of $\mathrm{P}(3 \mathrm{BCMU})$ is blue indicating a different electronic state of the conjugated backbone. Gels of $\mathrm{P}(4 \mathrm{BCMU})$ in $p$-DCB/TOL mixed solvent exhibit the gel-to-sol transition around $345 \mathrm{~K}$ and those of $\mathrm{P}(3 \mathrm{BCMU})$ around $387 \mathrm{~K}^{3}$ The transition temperatures of these gels are almost independent of polymer concentration and weakly depend on solvent quality and molecular weight. ${ }^{1-3}$

* Present address: Osaka Research Institute, Mitsui Toatsu Chemicals Inc., Takasago, Takaishi, Osaka, 592 Japan.

* To whom correspondence should be addressed. 
The alternating current (AC) conductivity $\sigma_{\mathrm{ac}}$ in the gel state is higher than that in the sol state. The enhancement of $\sigma_{\mathrm{ac}}$ by a dopant iodine and the photoconduction can be observed. Thus the conduction mechanism in $\mathrm{P}(3 \mathrm{BCMU})$ and $\mathrm{P}(4 \mathrm{BCMU})$ gels is mainly electronic rather than ionic.

In our preliminary study we found that $\mathrm{P}(3 \mathrm{BCMU})$ and $\mathrm{P}(4 \mathrm{BCMU})$ are miscible in a mixed solvent $p$-DCB/TOL above $385 \mathrm{~K}$. On cooling, the solution transformed into a gel having no turbidity. This indicates that both components are miscible even in the gel state at least in the macroscopic scale. In this paper we report the effects of blending on the electric conductivity, sol-gel transition and UV spectra in such blend gels.

There were two objectives. One was to investigate the effect of blending on the gelto-sol transition temperature. In gels of pure $\mathrm{P}(3 \mathrm{BCMU})$ and $\mathrm{P}(4 \mathrm{BCMU})$, the conformational transformation from rod to random coil occurres at the gel-to-sol transition temperature indicating that the confomational change and the formation of gel network are closely correlated. ${ }^{1,3}$ It is interesting to see at what temperature the $\mathrm{P}(3 \mathrm{BCMU})$ and $\mathrm{P}(4 \mathrm{BCMU})$ molecules transform from rod to coil in blend gels. For this purpose, measurements of shear moduli $G^{\prime}$ and UV spectra have been made.

The second objective was to examine the electric conductivity and the electronic state of $\mathrm{P}(3 \mathrm{BCMU})$ and $\mathrm{P}(4 \mathrm{BCMU})$ in the blends. Although a vast number of studies on the electronic conduction of conjugated polymers have been reported, ${ }^{10}$ there are few reports on conductivity in polymer blends consisting of two chemically different conducting polymers. The influence of blending on the conductivity in blend gels has been studied by comparing the behavior of blend gel with that of the pure gels reported previously.

\section{EXPERIMENTAL}

\section{Samples}

The details of preparation of $\mathrm{P}(4 \mathrm{BCMU})$ and $\mathrm{P}(3 \mathrm{BCMU})$ polymers were reported previously. ${ }^{6,7} \mathrm{P}(4 \mathrm{BCMU})$ with a weight average molecular weight $M_{w}$ of $4.5 \times 10^{5}$, and $\mathrm{P}(3 \mathrm{BCMU})$ with $M_{w}$ of $4.0 \times 10^{5}$ were used. Mixed solvent composed of $p$-dichlorobenzene ( $p$-DCB) and toluene (TOL) with a mixing ratio of $62 / 38$ (by weight) was dried with calcium hydride and filtered with teflon millipore film of $0.2 \mu \mathrm{m}$ mesh.

The gel samples were prepared as follows. First $\mathrm{P}(3 \mathrm{BCMU})$ and $\mathrm{P}(4 \mathrm{BCMU})$ were dissolved in $p$-DCB/TOL(62/38) with a prescribed mixing ratio. Then the system was heated to $390 \mathrm{~K}$ followed by stiring until it became a homogeneous solution. By cooling, a gel was formed accompanied by color change from yellow to purple. We observed blend gels with an optical microscope but did not find any macroscopic phase separation.

\section{Method}

The cell used for electrical measurement was the condenser cell reported previously. ${ }^{1}$ The electrodes had an area of $5 \mathrm{~cm}^{2}$ and about $0.2 \mathrm{~mm}$ separated. AC conductivities $\sigma_{a c}$ were measured with an automatic capacitance bridge (Yokogawa-Hewlett-Packard 4270A) at $5 \mathrm{~V}$ in the frequency range from 1 to $100 \mathrm{kHz}$. All conductivity measurements were made under an argon atmosphere at a heating rate of ca. $0.3 \mathrm{Kmin}^{-1}$.

UV absorption spectra were obtained with a spectrometer (Shimadzu model UV-200). The temperature dependence of absorbance was measured using monochromatized (530 $\mathrm{nm})$ light from a high power xenon lamp (Nihon Bunko Co., model LH-X500). ${ }^{1}$ The transmitted light through the sample sealed in an ampule was measured with a photodiode.

Elastic moduli $G^{\prime}$ of gels were measured with a coaxial cylinder type rheometer (Iwamoto Seisakusho Co., Autoviscometer IR-200). 


\section{RESULTS AND DISCUSSION}

\section{Gel-to-Sol Transition Temperature and Shear Moduli}

Figure 1 shows the temperature dependence of shear moduli $G^{\prime}$ of blend gels in which total polymer concentration is $0.50 \mathrm{wt} \%$. The weight fractions $w_{3}$ of $\mathrm{P}(3 \mathrm{BCMU})$ in the total polymer are 0.35 and 0.61 . The solid line indicates $G^{\prime}$ of pure $\mathrm{P}(3 \mathrm{BCMU})$ gel in the mixed solvent. The dash-dot line shows the $G^{\prime}$ of pure $\mathrm{P}(4 \mathrm{BCMU})$ gel estimated from the tensile modulus $E$ as follows. First $E$ for a gel of $\mathrm{P}(4 \mathrm{BCMU}) /[\mathrm{p}-\mathrm{DCB} / \mathrm{TOL}(62 / 38)]$ was determined to be $1.50 \times 10^{6} \mathrm{dyn} \cdot \mathrm{cm}^{-2}$ at concentration $C$ of $4.6 \mathrm{wt} \%$ by the method reported previously. ${ }^{2}$ Then the value was converted to $G^{\prime}$ at $0.50 \mathrm{wt} \%$ using the relation $E=3 G^{\prime}$ and assuming $E \propto C$. $^{2}$

It is noted that the $\mathrm{P}(4 \mathrm{BCMU}) /[\mathrm{p}-\mathrm{DCB} /$ TOL(62/38)] system is in the sol state above $345 \mathrm{~K}$ and hence $G^{\prime}$ vanishes above $c a .340 \mathrm{~K}$. We see that $G^{\prime}$ of blend gels are intermediate of those of pure $\mathrm{P}(4 \mathrm{BCMU})$ and $\mathrm{P}(3 \mathrm{BCMU})$ in the range of $T<340 \mathrm{~K}$. If we assume the

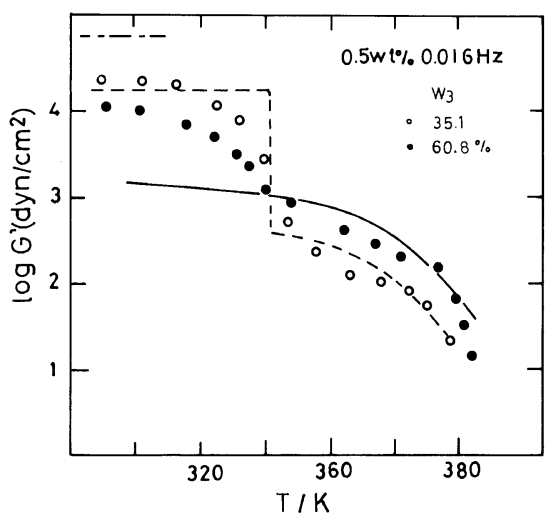

Figure 1. Temperature dependence of storage shear moduli $G^{\prime}$ at $0.016 \mathrm{~Hz}$ of blend gel with polymer concentration of $0.5 \mathrm{wt} \%$ and blend ratio $w_{3}$ of $\mathrm{P}(3 \mathrm{BCMU})$ against the total polymer is 0.351 and 0.608 . The dash-dot line and solid line indicate $G^{\prime}$ of pure $\mathrm{P}(4 \mathrm{BCMU})$ and $\mathrm{P}(3 \mathrm{BCMU})$ gel, respectively. The dashed line represents $G^{\prime}$ calculated from observed moduli of components by assuming additivity. additivity of $G^{\prime}$, the values of $\log G^{\prime}$ of the blend gels at $w_{3}=0.35$ and 0.61 become 4.2 and 3.9 , respectively at $300 \mathrm{~K}$ which agree approximately with the observed $G^{\prime}$ as shown in Figure 1. Therefore, we conclude that the network below $340 \mathrm{~K}$ is composed of both the $\mathrm{P}(3 \mathrm{BCMU})$ and $\mathrm{P}(4 \mathrm{BCMU})$ molecules.

The shear modulus $G^{\prime}$ of blend gels changes stepwise around $340 \pm 3 \mathrm{~K}$ which corresponds to the gel-to-sol transition temperature $T_{\mathrm{tr}}(4)$ ( $=345 \mathrm{~K})$ of pure $\mathrm{P}(4 \mathrm{BCMU})$ gel. On further heating, the gels transform completely to sols at $385 \pm 3 \mathrm{~K}$ which corresponds to the transition temperature $T_{\mathrm{tr}}(3)(=387 \mathrm{~K})$ of pure $\mathrm{P}(3 \mathrm{BCMU})$ gel. These transition temperatures are slightly lower than those of pure gels. In the range of $T_{\mathrm{tr}}(4)<T<T_{\mathrm{tr}}(3)$, observed $G^{\prime}$ is approximately equal to $w_{3}$ times the $G^{\prime}$ of pure $\mathrm{P}(3 \mathrm{BCMU})$ as indicated by the dashed line for the gel of $w_{3}=0.35$. Thus, the $\mathrm{P}(4 \mathrm{BCMU})$ molecules do not contribute to elasticity in the range of $T>T_{\mathrm{tr}}(4)$.

Network structures below and above $T_{\mathrm{tr}}(4)$ are speculated as shown schematically in Figures $2 \mathrm{a}$ and $2 \mathrm{~b}$, where the thick and thin lines indicate $\mathrm{P}(4 \mathrm{BCMU})$ and $\mathrm{P}(3 \mathrm{BCMU})$, respectively. In the range of $T<T_{\mathrm{tr}}(4)$, the network of the blend gel is formed by both the rod-like $\mathrm{P}(3 \mathrm{BCMU})$ and $\mathrm{P}(4 \mathrm{BCMU})$ molecules. Two types of cross-links are considered. One is linkage between molecules of the same kind and the other is between the $\mathrm{P}(3 \mathrm{BCMU})$ and $\mathrm{P}(4 \mathrm{BCMU})$ molecules. It is difficult, however, to conclude which or whether both
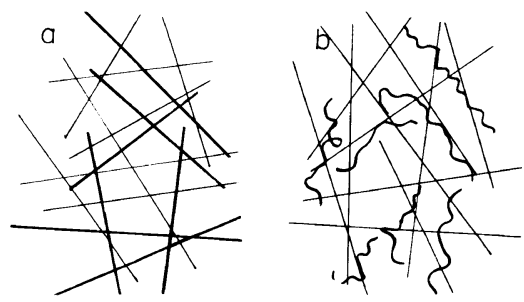

Figure 2. Schematic representation of the structure of blend gel in the temperature $T$ ranges of (a) $T<T_{\text {tr }}$ (4) and (b) $T_{\text {tr }}(4)<T<T_{\text {tr }}(3)$. Thick and thin lines indicate $\mathrm{P}(4 \mathrm{BCMU})$ and $\mathrm{P}(3 \mathrm{BCMU})$, respectively. 
are formed. Above $T_{\mathrm{tr}}(4)$, only the $\mathrm{P}(3 \mathrm{BCMU})$ molecules preserve the rod-like conformation and form a network but $\mathrm{P}(4 \mathrm{BCMU})$ molecules with the random coil conformation locate in the mesh as shown in Figure 2b. Probably crosslinks between the rod-like $\mathrm{P}(3 \mathrm{BCMU})$ molecules and coil-like $\mathrm{P}(4 \mathrm{BCMU})$ molecules may be formed by hydrogen bonding of the urethane groups ${ }^{11}$ but the $\mathrm{P}(4 \mathrm{BCMU})$ chains do not contribute to the $G$ ' since the entropy elasticity of the flexible chains is much smaller than the energetic (enthalpy) elasticity due to bending of the rod-like P(3BCMU) molecules.

\section{Electric Conductivity}

In Figure 3, $\sigma_{\mathrm{ac}} v s$. temperature curves for the blend gels with various $\mathrm{P}(3 \mathrm{BCMU})$ contents are shown. Total polymer concentra-

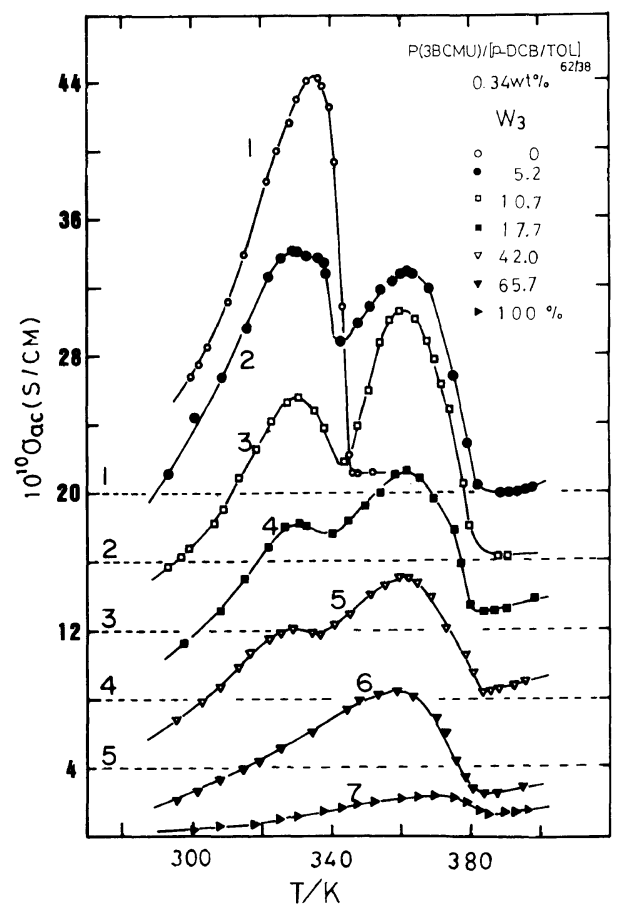

Figure 3. Temperature dependence of alternating current conductivity $\sigma_{\mathrm{ac}}$ at $1 \mathrm{kHz}$ for blend gels with total polymer concentration of $0.34 \mathrm{wt} \%$. The weight fraction $w_{3}$ of $\mathrm{P}(3 \mathrm{BCMU})$ in the total polymer is indicated in the figure. Curves 1 to 5 have been shifted upward. The dashed lines indicate the base lines for the $\sigma_{\mathrm{ac}}$ curves of the same number. tion was fixed at $0.345 \mathrm{wt} \%$. The data for pure $\mathrm{P}(4 \mathrm{BCMU})$ and $\mathrm{P}(3 \mathrm{BCMU})$ gels in the same mixed solvent are also shown.

It is seen that pure $\mathrm{P}(4 \mathrm{BCMU})$ and $\mathrm{P}(3 \mathrm{BCMU})$ gels exhibit a single peak around 330 and $360 \mathrm{~K}$, respectively. Above the each peak temperature, $\sigma_{\mathrm{ac}}$ decreases steeply and then becomes minimum at the gel-to-sol transition temperature $T_{\mathrm{r}}: T_{\mathrm{tr}}(4)=345 \mathrm{~K}$ and $T_{\mathrm{tr}}(3)=387 \mathrm{~K}$. In our previous paper we demonstrated that the peak of $\sigma_{\mathrm{ac}}$ in pure $\mathrm{P}(3 \mathrm{BCMU})$ and $\mathrm{P}(4 \mathrm{BCMU})$ corresponds to the incipience of the gel-to-sol transition and above the peak temperature the network structure degrades with increasing temperature. At temperatures of minimum $\sigma_{\mathrm{ac}}$, the gels transform completely to sols. ${ }^{1,3}$

In the blend gels, two peaks are seen at temperatures corresponding to the peak temperatures of the pure gels. We see that the first peak around $330 \mathrm{~K}$ decreases with increasing $\mathrm{P}(3 \mathrm{BCMU})$ content indicating that the peak is due to $\mathrm{P}(4 \mathrm{BCMU})$. On the other hand, the intensity of the second peak around $360 \mathrm{~K}$ shows a peculiar $w_{3}$ dependence. Figure 4 shows the $w_{3}$ dependence of $\sigma_{\mathrm{ac}}$ at 330 and $360 \mathrm{~K}$. We see that $\sigma_{\mathrm{ac}}$ at $330 \mathrm{~K}$ near the first peak decreases approximately in proportion to $w_{3}$. The conductivity $\sigma_{\text {ac }}$ at $360 \mathrm{~K}$ around the second peak increases steeply in the range of $w_{3}<0.1$ and decreases gradually beyond $w_{3}=0.1$.

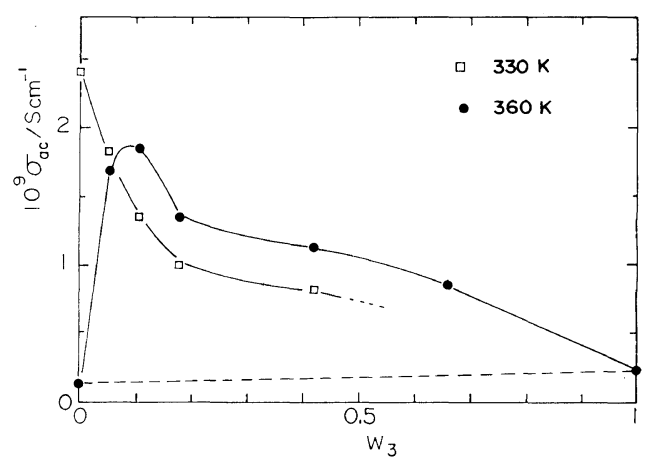

Figure 4. $\sigma_{\mathrm{ac}}$ at $330 \mathrm{~K}$ and $360 \mathrm{~K}$ plotted against the weight fraction $w_{3}$ of $\mathrm{P}(3 \mathrm{BCMU})$ in the total polymer. The dashed line indicates $\sigma_{\mathrm{ac}}$ at $360 \mathrm{~K}$ expected from additivity. 

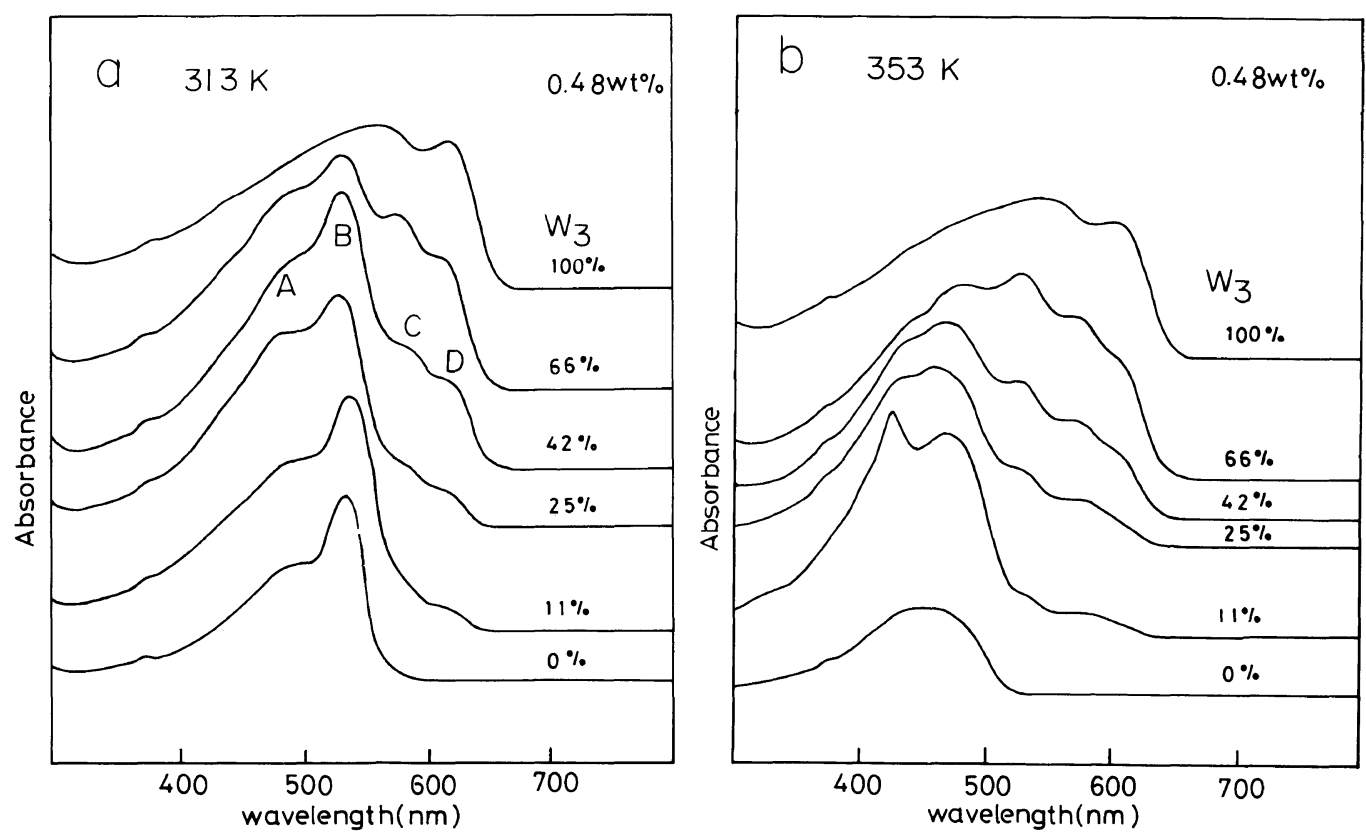

Figure 5. Ultraviolet and visible spectra of blend gels at (a) $313 \mathrm{~K}$ and (b) $353 \mathrm{~K}$ for gels with various $\mathrm{P}(3 \mathrm{BCMU})$ content $w_{3}$. Total polymer concentration is $0.48 \mathrm{wt} \%$. Base lines have been shifted. Absorbance around $700 \mathrm{~nm}$ indicates approximately zero absorbance.

We recognize that in the temperature range $T_{\mathrm{tr}}(4)<T<T_{\mathrm{tr}}(3), \sigma_{\mathrm{ac}}$ deviates strongly from additivity as typically shown by the dashed line in Figure 4. The level of $\sigma_{\mathrm{ac}}$ of the blend is much higher than those of the components. We speculate that the coil-like $\mathrm{P}(4 \mathrm{BCMU})$ molecules act as a dopant against the $\mathrm{P}(3 \mathrm{BCMU})$ molecules and that a charge transfer complex is formed partially between the $\mathrm{P}(3 \mathrm{BCMU})$ and $\mathrm{P}(4 \mathrm{BCMU})$ chains.

\section{UV Spectra and Color Change}

The UV spectra of blend gels at $313 \mathrm{~K}$ and $353 \mathrm{~K}$ are shown in Figures $5 \mathrm{a}$ and $5 \mathrm{~b}$, respectively, in which the polymer concentration was fixed at $0.48 \mathrm{wt} \%$ and the blend ratio changed widely. For comparison, the spectra of pure $\mathrm{P}(3 \mathrm{BCMU})$ and $\mathrm{P}(4 \mathrm{BCMU})$ are also shown. As mentioned above, both the $\mathrm{P}(3 \mathrm{BCMU})$ and $\mathrm{P}(4 \mathrm{BCMU})$ chains contribute to elasticity at $313 \mathrm{~K}$ but only $\mathrm{P}(3 \mathrm{BCMU})$ does so at $353 \mathrm{~K}$.

The spectrum of the blend gel at $313 \mathrm{~K}$ con- sists of four absorption bands at 470 (A), 530 (B), 560 (C), and $630 \mathrm{~nm}$ (D). The bands A and $\mathrm{B}$ are seen in pure $\mathrm{P}(4 \mathrm{BCMU})$ gel and hence are due to $P(4 B C M U)$ but $C$ and $D$ are due to $\mathrm{P}(3 \mathrm{BCMU})$. Bands $\mathrm{B}$ and $\mathrm{D}$ were previously assigned to photo excited $\pi-\pi *$ excitons along the extended rod-like chains for $\mathrm{P}(3 \mathrm{BCMU})$ and $\mathrm{P}(4 \mathrm{BCMU})$ molecules, respectively. ${ }^{12}$ Bands $A$ and $C$ are due to the $\pi-\pi^{*}$ transition of the coiled chains.

In the pure $\mathrm{P}(4 \mathrm{BCMU})$ system, band $\mathrm{B}$ disappears completely at $353 \mathrm{~K}$ since it is in the sol state in which the $\mathrm{P}(4 \mathrm{BCMU})$ chains assume random coil conformation $\left(T_{\mathrm{tr}}(4)=345 \mathrm{~K}\right)$. However in blend gels, this band still remains at $353 \mathrm{~K}$ as shown in Figure $5 \mathrm{~b}$ indicating that the rod-like parts still exist even above $T_{\text {tr }}(4)$.

The difference in the spectra at $353 \mathrm{~K}$ between blend gels and a hypothetical gel in which the $\mathrm{P}(3 \mathrm{BCMU})$ and $\mathrm{P}(4 \mathrm{BCMU})$ molecules do not interact each other was estimated as follows. First, we calculated hypothetical absorbance $\alpha_{\text {calc }}$ from the absorbances of pure 


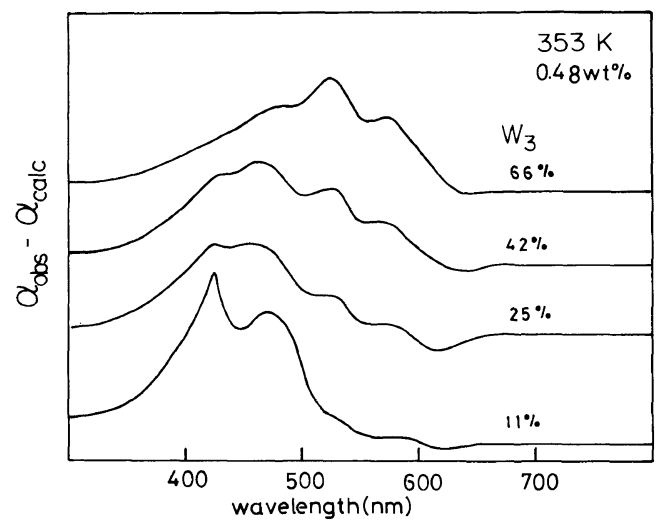

Figure 6. Difference of absorbance $\alpha_{\text {obs }}$ of the blend gel and calculated absorbance $\alpha_{\text {calc }}$ by assuming additivity at $353 \mathrm{~K}$.

$\mathrm{P}(3 \mathrm{BCMU})$ gel and $\mathrm{P}(4 \mathrm{BCMU})$ sol at $353 \mathrm{~K}$ by assuming additivity. Then $\alpha_{\text {calc }}$ was subtracted from the observed absorbance $\alpha_{\text {obs}}$. The result is shown in Figure 6. We see that the intensity of the band at $530 \mathrm{~nm}$ (peak B) increases with the $\mathrm{P}(3 \mathrm{BCMU})$ content. In addition, a relatively sharp band not seen in the pure gels is seen at $425 \mathrm{~nm}$. We cannot assign this band at the present stage. However, this indicates that the electronic states of the $\mathrm{P}(3 \mathrm{BCMU})$ and $\mathrm{P}(4 \mathrm{BCMU})$ molecules differ from the pure state and this is consistent with the speculation that a charge transfer complex is formed partly between these molecules.

In order to investigate the conformational change of $\mathrm{P}(4 \mathrm{BCMU})$ molecules in more detail, we measured the temperature dependence of transmittance $\tau$ at $530 \mathrm{~nm}$. It is noted that the band at this wave length reflects a conjugated electronic state. Figure 7 shows plots of $\tau v s$. $T$ for $0.5 \mathrm{wt} \%$ gels with several $\mathrm{P}(3 \mathrm{BCMU})$ contents. In the range of $\mathrm{P}(3 \mathrm{BCMU})$ content below $30 \%$, there is a sharp increase of the transmittance at $340 \mathrm{~K}$ as seen for the pure $\mathrm{P}(4 \mathrm{BCMU})$ gel. This is obviously due to the partial rod-to-coil transition of the $\mathrm{P}(4 \mathrm{BCMU})$ chains. However, in the blend gels above $340 \mathrm{~K}$, transmittance is much lower than the pure $\mathrm{P}(4 \mathrm{BCMU})$ gel reflecting the presence of band $B$. Transmit-

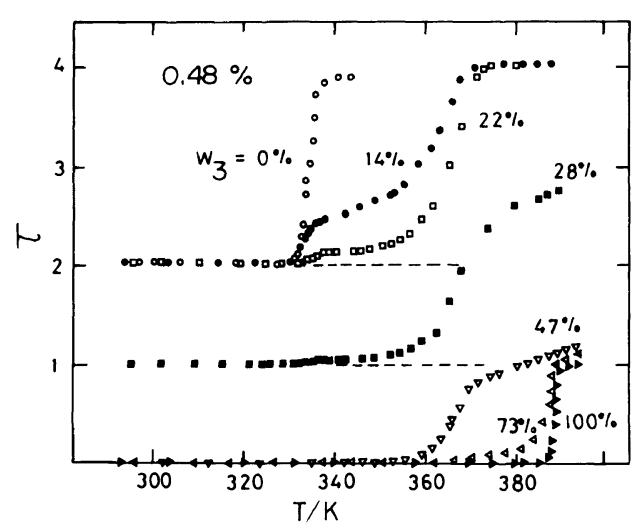

Figure 7. Temperature dependence of transmittance in an arbitrary scale at $530 \mathrm{~nm}$ for blends with various $\mathrm{P}(3 \mathrm{BCMU})$ content $w_{3}$. Total polymer concentration is $0.48 \mathrm{wt} \%$. The base lines have been shifted upward as indicated by the dashed lines.

tance gradually increases with temperature and reaches the level of pure $\mathrm{P}(4 \mathrm{BCMU})$ around $370 \mathrm{~K}$. At higher concentrations the transition at $340 \mathrm{~K}$ becomes less clear.

In pure $\mathrm{P}(3 \mathrm{BCMU})$, transmittance changes stepwise at $385 \mathrm{~K}$ but in blend gels transmittance increases rather gradually in the range of 360 to $380 \mathrm{~K}$. This indicates that in blend gels, the $\mathrm{P}(3 \mathrm{BCMU})$ molecules commence to transform from rod to coil at lower temperature than the pure $\mathrm{P}(3 \mathrm{BCMU})$ gel. This suggests that interaction between the $\mathrm{P}(4 \mathrm{BCMU})$ and $\mathrm{P}(3 \mathrm{BCMU})$ molecules, which has been assumed in the former section, weakens the network of the gel.

\section{CONCLUSIONS}

1. In the blend gel of $\mathrm{P}(3 \mathrm{BCMU})$ and $\mathrm{P}(4 \mathrm{BCMU})$, both components contribute to elasticity in the range of $T<340 \mathrm{~K}$, but only $\mathrm{P}(3 \mathrm{BCMU})$ contributes in the range of $340<$ $T<385 \mathrm{~K}$. UV spectra indicate that the conformation of the $\mathrm{P}(4 \mathrm{BCMU})$ molecules in blend gels changes from rod to random coil at $340 \mathrm{~K}$ but conformation of the P(3BCMU) molecules changes at $385 \mathrm{~K}$.

2. Two maxima are observed in the 
alternating current conductivity $\sigma_{\mathrm{ac}}$ vs. $T$ curves of blend gels at 330 and $360 \mathrm{~K}$ which correspond to $\sigma_{\mathrm{ac}}$ maximum temperatures of pure $\mathrm{P}(4 \mathrm{BCMU})$ and $\mathrm{P}(3 \mathrm{BCMU})$ gels, respectively.

3. In the temperature range of $340<T<$ $385, \sigma_{\mathrm{ac}}$ is $c a .10$ times higher than the value expected from $\sigma_{\mathrm{ac}}$ of the components. In this range, UV spectra indicate the presence of the rod-like conformation of the $\mathrm{P}(4 \mathrm{BCMU})$ chains. A new band not seen in pure gels is also observed. There is the possibility that a charge transfer complex is formed between the $\mathrm{P}(3 \mathrm{BCMU})$ and $\mathrm{P}(4 \mathrm{BCMU})$ molecules.

\section{REFERENCES}

1. P. Chen, K. Adachi, and T. Kotaka, Polymer, 33,
1363 (1992).

2. P. Chen, K. Adachi, and T. Kotaka, Polymer, 33, 1813 (1992).

3. P. Chen, K. Adachi, and T. Kotaka, Polym. J., 24, 1025 (1992).

4. G. N. Patel and E. K. Walsh, J. Chem. Phys., 70, 4387 (1979).

5. R. R. Chance, G. N. Patel, and J. D. Witt, J. Chem. Phys., 71, 206 (1979).

6. K. Se, H. Ohnuma, and T. Kotaka, Polym. J., 14, 895 (1982).

7. K. Se, H. Ohnuma, and T. Kotaka, Macromolecules, 16, 1581 (1983).

8. H. Ohnuma, K. Inoue, K. Se, and T. Kotaka, Macromolecules, 17, 1285 (1984).

9. G. N. Patel, J. D. Witt, and Y. P. Khanna, J. Polym. Sci., Polym. Phys. Ed., 18, 1383 (1980).

10. For example papers presented in Synthetic Metals, 1, 99 (1980).

11. P. Chen, K. Adachi, and T. Kotaka, in preparation.

12. T. Kanetake, T. Yoshinori, T. Koda, T. Kotaka, and H. Ohnuma, J. Phys. Soc. Jpn., 54, 4014 (1985). 